BMJ Open

Diabetes

Research

\& Care

\section{Association between hemoglobin A1c variability and hypoglycemia-related hospitalizations in veterans with diabetes mellitus}

\author{
Molly J Y Zhao (D) ,1,2 Julia C Prentice, , ${ }^{1,3}$ David C Mohr, ${ }^{1,4}$ Paul R Conlin ${ }^{5,6}$
}

To cite: Zhao MJY, Prentice JC, Mohr DC, et al. Association between hemoglobin A1C variability and hypoglycemiarelated hospitalizations in veterans with diabetes mellitus. BMJ Open Diab Res Care 2021;9:e001797. doi:10.1136/ bmjdrc-2020-001797

- Supplemental material is published online only. To view please visit the journal online (http://dx.doi.org/10.1136/ bmjdrc-2020-001797).

Received 25 July 2020 Revised 10 November 2020 Accepted 14 December 2020

\section{Check for updates}

(C) Author(s) (or their employer(s)) 2021. Re-use permitted under CC BY-NC. No commercial re-use. See rights and permissions. Published by BMJ.

For numbered affiliations see end of article.

Correspondence to

Molly J Y Zhao; mollyjz@bu.edu

\section{ABSTRACT}

Introduction To study the impact of hemoglobin A1c (A1c) variability on the risk of hypoglycemia-related hospitalization $(\mathrm{HRH})$ in veterans with diabetes mellitus. Research design and methods 342059 veterans with diabetes aged 65 years or older were identified for a retrospective cohort study. All participants had a 3-year baseline period from January 1, 2005 to December 31, 2016, during which they had at least four A1c tests. A1C variability measures included coefficient of variation (A1C CV), A1c SD, and adjusted A1c SD. HRH was identified during a 2-year follow-up period from Medicare and the Veterans Health Administration through validated algorithms of International Classification of Diseases (ICD)9 and ICD-10 codes. Logistic regression modeling was used to evaluate the relationship between $\mathrm{A} 1 \mathrm{c}$ variability and $\mathrm{HRH}$ risk while controlling for relevant clinical covariates.

Results 2871 patients had one or more HRH in the 2year follow-up period. HRH risk increased with greater A1c variability, and this was consistent across A1c CV, A1C SD, and adjusted A1c SD. Average A1c levels were also independently associated with $\mathrm{HRH}$, with levels $<7.0 \%$ (53 mmol $/ \mathrm{mol}$ ) having lower risk and >9\% (75 mmol/mol) with greater risk. The relationships between $\mathrm{A} 1 \mathrm{c}$ variability remained significant after controlling for average A1C levels and prior HRH during the baseline period. Conclusion Increasing A1c variability and elevated A1c levels are associated with a greater risk of $\mathrm{HRH}$ in older adults with diabetes. Clinicians should consider A1c variability when assessing patients for risk of severe hypoglycemia.

\section{INTRODUCTION}

Severe hypoglycemia resulting in hospitalization leads to poor health outcomes and mortality in older adults with diabetes mellitus. $^{1-5}$ Concerns about treatmentassociated hypoglycemia have assumed greater importance as rates of hypoglycemiarelated hospitalization (HRH) increased between 1999 and 2011 and surpassed hyperglycemia-related hospitalization rates between 1999 and 2011. ${ }^{6}$

Several patient-level risk factors independently predict severe hypoglycemia

\section{Significance of this study}

What is already known about this subject?

- Hypoglycemia-related hospitalization (HRH) increases the risk of mortality in older adults with diabetes.

- Several patient-level factors predict the risk of severe hypoglycemia, but hemoglobin A1c (A1c) levels have an uncertain relationship to $\mathrm{HRH}$, which highlights that A1c levels alone may be insufficient to understand risk.

- Variability in A1c levels is associated with increased risk of diabetes complications and mortality.

What are the new findings?

- Increasing A1c variability was associated with greater risk of HRH over a 2-year follow-up period, after controlling for A1c levels and several clinical and sociodemographic covariates.

- Higher A1c levels $>9 \%(75 \mathrm{mmol} / \mathrm{mol})$ conferred greater risk of $\mathrm{HRH}$ after controlling for $\mathrm{A} 1 \mathrm{c}$ variability.

- The relationship between A1c variability and $\mathrm{HRH}$ risk remained significant after controlling for prior HRH events.

How might these results change the focus of research or clinical practice?

- A1c variability over time should be considered when assessing risk of severe hypoglycemia in older adults with diabetes.

events, such as older age, diabetes treatment that includes insulin or sulfonylureas, black race, lower body mass index (BMI), renal disease, cognitive impairment, and history of hypoglycemic events. ${ }^{2}$ 7-10 Additional concerns exist for older adults who are potentially overtreated in the setting of comorbid conditions. ${ }^{11}$ Thus, many diabetes treatment guidelines favor individualized and higher hemoglobin A1c (A1c) targets for at-risk older adults to balance long-term glycemic benefits and short-term hypoglycemia risk. ${ }^{12-15}$ Maintaining patients in an appropriate glycemic range is also complicated by uncertainty about the relationship between A1c and risk 
of hypoglycemia. Some studies show that higher A1c confers increased risk of hypoglycemia, ${ }^{16}$ while others show an inverse relationship, with lower A1c associated with increased risk. ${ }^{17}$ This suggests that A1c levels alone may not define risk but are part of a dynamic relationship with patient-level factors and medications that result in greater glucose variability over time.

A1c variability is associated with increased hospitalizations, diabetes complications, and mortality. ${ }^{18-23}$ These risks persist when controlled for A1c levels ${ }^{18} 1921$ and are independent of standard or intensive diabetes treatment. ${ }^{24}{ }^{25}$ Therefore, more indepth study of the relationship between Alc variability and HRH is warranted.

This study was designed to validate the clinical implications of A1c variability and substantiate its effects on HRH in older adults with diabetes. We used a large nationwide sample of veterans with diabetes to study the association between measures of A1c variability and risk of HRH while controlling for several relevant sociodemographic and clinical factors.

\section{METHODS}

\section{Study population}

We combined administrative data sets from the Veterans Health Administration (VA) and Medicare to gather sociodemographic and clinical measures and outpatient and inpatient utilization. Visit dates and diagnosis codes necessary for identifying HRH were obtained from inpatient discharge records in VA and Medicare inpatient databases. Medications, laboratory tests, financial means tests, and percentage of service-connected disability were extracted from the VA's administrative claims.

We identified veterans diagnosed with diabetes who were aged 65 years or older, enrolled in VA care and dually eligible for Medicare during the period of January 1, 2005 through December 31, 2014 (figure 1). A diabetes diagnosis was determined using published criteria ${ }^{26}$ : (1) two or more diabetes diagnosis codes from outpatient visits or (2) one inpatient hospitalization for diabetes over a 2-year period or (3) a prescription for diabetes medication (excluding metformin alone) in the current year.

Patients meeting the following criteria:

a) VA-Medicare eligible patients

b) Two outpatient visits or one inpatient visit with an ICD-9 code for diabetes (362.0X, $357.2,250 . X, 366.41$ ), or prescription for a diabetes medication (excluding metforminonly)

c) $\geq 4$ Alc tests in a 3-year baseline period between 2005 and 2013

Excluded patients younger than 65 in the year prior to baseline date

Excluded patients without a full 3-year baseline period

Excluded patients with missing comorbidities

Excluded patients whose 3-year baseline period ended after 2014

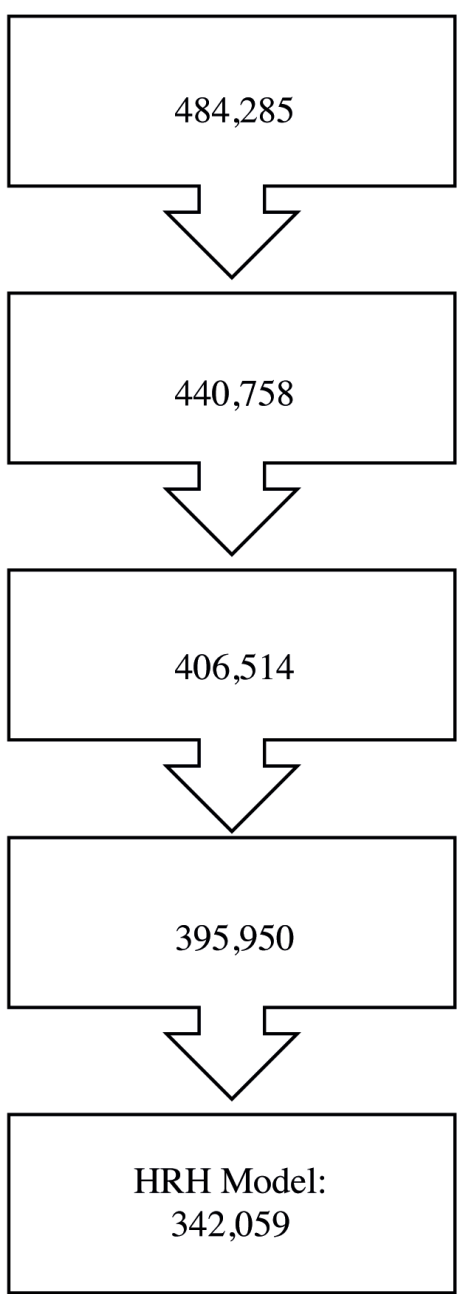

Excluded patients who died anytime in the twoyear follow up period

Figure 1 Flow chart of the selective criteria used to create the final study sample ( $\mathrm{N}=342$ 059). HRH, hypoglycemia-related hospitalization; ICD, International Classification of Diseases; VA, Veterans Health Administration. 
Table 1 Sociodemographic and clinical characteristics

\begin{tabular}{|c|c|c|c|c|c|c|c|}
\hline & \multicolumn{2}{|c|}{$\begin{array}{l}\text { Non-HRH population } \\
(n=339188)\end{array}$} & \multicolumn{2}{|c|}{$\begin{array}{l}\text { HRH population } \\
(\mathrm{n}=2871)\end{array}$} & \multicolumn{2}{|c|}{$\begin{array}{l}\text { Study population } \\
(\mathrm{N}=342059)\end{array}$} & \multirow[b]{2}{*}{$P$ value } \\
\hline & Patients (n) & $\%$ & Patients (n) & $\%$ & Patients (n) & $\%$ & \\
\hline Sex & & & & & & & 0.325 \\
\hline Male & 334814 & 99 & 2828 & 99 & 337642 & 99 & \\
\hline Female & 4374 & 1 & 43 & 1 & 4417 & 1 & \\
\hline Race & & & & & & & $<0.001$ \\
\hline White & 292495 & 86 & 2162 & 75 & 294657 & 86 & \\
\hline Black & 36307 & 11 & 612 & 21 & 36919 & 11 & \\
\hline Hispanic & 5151 & 2 & 55 & 2 & 5206 & 2 & \\
\hline Asian & 1283 & 0 & 14 & 0 & 1297 & 0 & \\
\hline Other & 3952 & 1 & 28 & 1 & 3980 & 1 & \\
\hline Age (years) & & & & & & & $<0.001$ \\
\hline $64-74$ & 203585 & 60 & 1332 & 46 & 204917 & 60 & \\
\hline $75+$ & 135603 & 40 & 1539 & 54 & 137142 & 40 & \\
\hline \multicolumn{8}{|l|}{ Diabetes medication use } \\
\hline Insulin & & & & & & & $<0.001$ \\
\hline No & 262008 & 77 & 1445 & 50 & 263453 & 77 & \\
\hline Yes & 77180 & 23 & 1426 & 50 & 78606 & 23 & \\
\hline Metformin & & & & & & & $<0.001$ \\
\hline No & 165771 & 49 & 1685 & 59 & 167456 & 49 & \\
\hline Yes & 173417 & 51 & 1186 & 41 & 174603 & 51 & \\
\hline Sulfonylurea & & & & & & & $<0.001$ \\
\hline No & 157968 & 47 & 1007 & 35 & 158975 & 47 & \\
\hline Yes & 181220 & 53 & 1864 & 65 & 183084 & 54 & \\
\hline Alpha-glucosidase inhibitor & & & & & & & 0.013 \\
\hline No & 332630 & 98 & 2797 & 97 & 335427 & 98 & \\
\hline Yes & 6558 & 2 & 74 & 3 & 6632 & 2 & \\
\hline Thiazolidinedione & & & & & & & $<0.001$ \\
\hline No & 283997 & 84 & 2258 & 79 & 286255 & 84 & \\
\hline Yes & 55191 & 16 & 613 & 21 & 55804 & 16 & \\
\hline Other medications* & & & & & & & 0.512 \\
\hline No & 333948 & 98 & 2831 & 99 & 336779 & 98 & \\
\hline Yes & 5240 & 2 & 40 & 1 & 5280 & 2 & \\
\hline Average A1c (\%) & & & & & & & $<0.001$ \\
\hline$<6$ & 38233 & 11 & 175 & 6 & 38408 & 11 & \\
\hline $6-6.9$ & 157548 & 46 & 878 & 31 & 158426 & 46 & \\
\hline $7-7.9$ & 97676 & 29 & 1015 & 35 & 98691 & 29 & \\
\hline $8-8.9$ & 32664 & 10 & 496 & 17 & 33160 & 10 & \\
\hline$\geq 9$ & 13067 & 4 & 307 & 11 & 13374 & 4 & \\
\hline Serum creatinine (mg/dL) & & & & & & & $<0.001$ \\
\hline$<0.6$ & 298 & 0 & 3 & 0 & 301 & 0 & \\
\hline $0.6-1.2$ & 188122 & 55 & 1027 & 36 & 189149 & 55 & \\
\hline$>1.2$ & 141253 & 42 & 1780 & 62 & 143033 & 42 & \\
\hline Missing† & 9515 & 3 & 61 & 2 & 9576 & 3 & \\
\hline Urine albumin to creatinine & ratio (mg/g) & & & & & & $<0.001$ \\
\hline
\end{tabular}

Continued 
Table 1 Continued

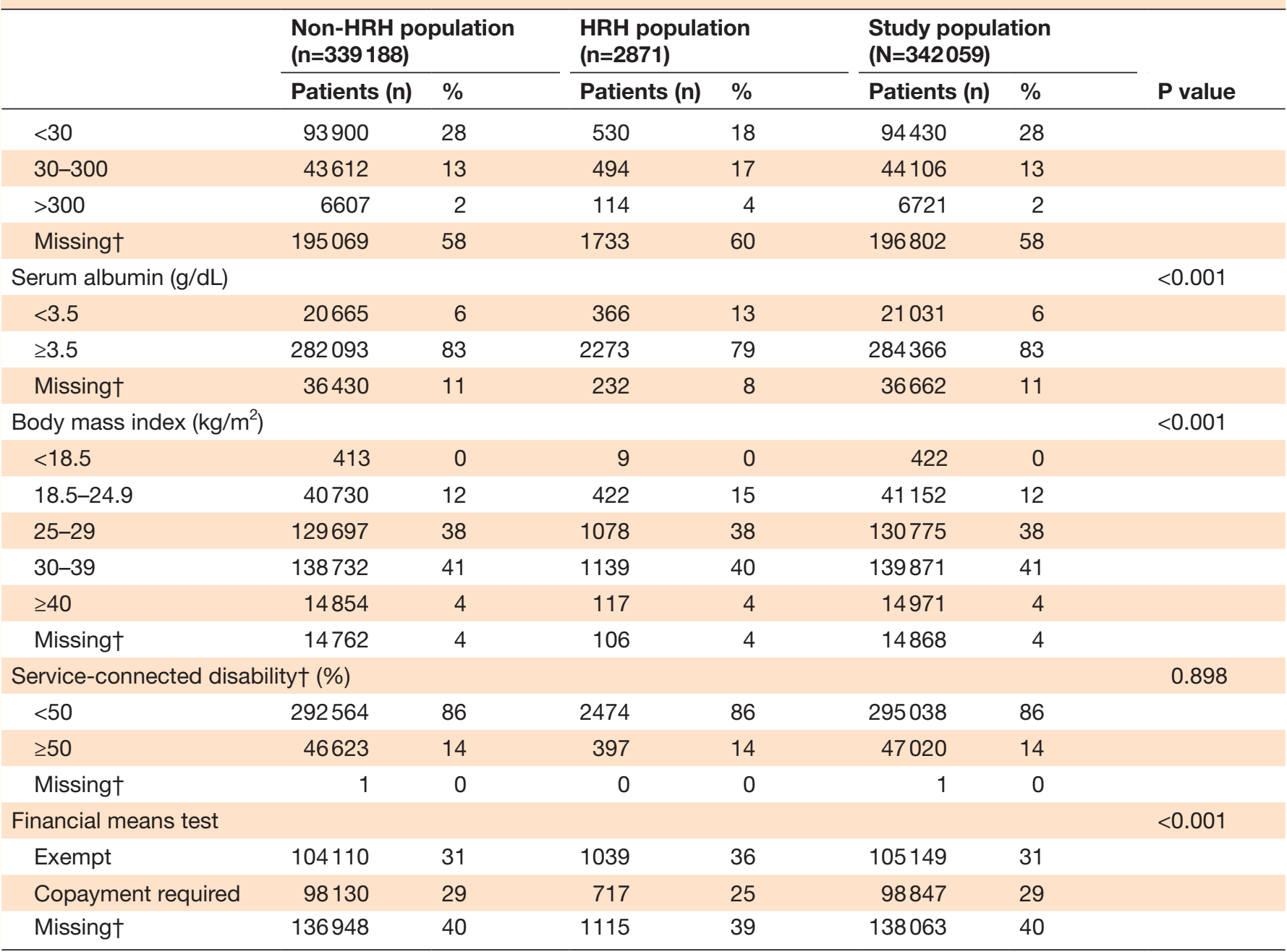

*Other medications: amylin analog, bile acid sequestrants, dipeptidyl peptidase inhibitors, dopamine receptor agonist, glucagon-like peptide, meglitinides, and sodium-glucose cotransporter inhibitor.

†Values missing from source database.

$\mathrm{HRH}$, hypoglycemia-related hospitalization.

Patients taking metformin alone were included if they had concomitant diabetes diagnosis codes. The latter typically captures at least $97 \%$ of patients with diabetes. ${ }^{26}$ A small number of patients may take metformin for nondiabetes diagnoses, so this criterion was used to increase specificity. Patients were required to have four or more A1c measurements over a consecutive 3-year baseline period, with sequential A1c tests $\leq 365$ days apart. A total of 395950 patients met these criteria. We excluded 53891 patients who died in the follow-up period. Thus, 342059 patients remained in the study sample for statistical analyses.

\section{Outcomes, exposures and covariates}

HRH was defined as hospital admissions with a principal discharge diagnosis of hypoglycemia based on validated algorithms of International Classification of Diseases (ICD)-9 and ICD-10 codes $^{27} 28$ occurring prior to December 31, 2016. The outcome did not include transfers and secondary diagnoses of hypoglycemia because these may have occurred during hospitalization or secondary to another acute event. ${ }^{6}$

A1c variability was described by A1c coefficient of variation (A1c CV), A1c SD, and adjusted A1c SD. A1c CV was calculated by dividing A1c SD by the average A1c value and expressed as per cent. Adjusted A1c SD accounted for the number of A1c measurements and the days between each measurement using a linear regression formula. ${ }^{21}$ Finally, the three measures of A1c variability were transformed into quartiles for analysis. We also included mean A1c categories $(<6 \%(42 \mathrm{mmol} / \mathrm{mol})$, $6 \%-6.9 \% \quad(42-52 \mathrm{mmol} / \mathrm{mol}), 7 \%-7.9 \% \quad(53-63 \mathrm{mmol} /$ $\mathrm{mol}), 8 \%-8.9 \% \quad(64-74 \mathrm{mmol} / \mathrm{mol}), \geq 9 \% \quad(75 \mathrm{mmol} /$ mol)) as a covariate to assess the independent effect of Alc variability on $\mathrm{HRH}$.

Sociodemographic factors included age at the start of the baseline period (categorized as $65-74$ and $\geq 75$ years), sex, race, financial means test (which assesses financial resources and determines a requirement for 
Table 2 Summary of A1c variability measures and HRH risk during 2-year follow-up period $(n=342058)^{*}$

\begin{tabular}{|c|c|c|}
\hline Modelt & OR $(95 \% \mathrm{Cl})$ & $P$ value \\
\hline \multicolumn{3}{|l|}{ Model 1} \\
\hline \multicolumn{3}{|c|}{ A1c coefficient of variation $(\%)($ ref $<4)$} \\
\hline $4-5.9$ & 1.19 (1.04 to 1.36$)$ & 0.011 \\
\hline $6-9.4$ & 1.28 (1.13 to 1.47$)$ & $<0.001$ \\
\hline $9.5-66$ & 1.44 (1.26 to 1.65$)$ & $<0.001$ \\
\hline \multicolumn{3}{|c|}{ A1c mean $(\%)(r e f=7-7.9)$} \\
\hline$<6$ & 0.66 (0.56 to 0.78$)$ & $<0.001$ \\
\hline $6-6.9$ & 0.78 (0.71 to 0.86$)$ & $<0.001$ \\
\hline $8-8.9$ & 1.11 (1.00 to 1.24$)$ & 0.060 \\
\hline$\geq 9$ & 1.53 (1.33 to 1.75$)$ & $<0.001$ \\
\hline \multicolumn{3}{|l|}{ Model 2} \\
\hline \multicolumn{3}{|c|}{ A1c SD (ref <0.25) } \\
\hline $0.25-0.40$ & 1.26 (1.10 to 1.45$)$ & 0.001 \\
\hline $0.41-0.68$ & 1.36 (1.18 to 1.56$)$ & $<0.001$ \\
\hline $0.69-6.46$ & 1.56 (1.35 to 1.81$)$ & $<0.001$ \\
\hline \multicolumn{3}{|c|}{ A1c mean $(\%)($ ref $=7-7.9)$} \\
\hline$<6$ & 0.71 (0.59 to 0.84$)$ & $<0.001$ \\
\hline $6-6.9$ & 0.81 ( 0.73 to 0.89$)$ & $<0.001$ \\
\hline $8-8.9$ & 1.09 (0.98 to 1.22$)$ & 0.125 \\
\hline$\geq 9$ & 1.49 (1.30 to 1.72$)$ & $<0.001$ \\
\hline \multicolumn{3}{|l|}{ Model 3} \\
\hline \multicolumn{3}{|c|}{ Adjusted A1c SD (ref <0.62) } \\
\hline $0.62-0.97$ & $1.08(0.95$ to 1.24$)$ & 0.239 \\
\hline $0.98-1.56$ & 1.12 (0.98 to 1.27$)$ & 0.103 \\
\hline $1.57-17.40$ & 1.37 (1.20 to 1.57$)$ & $<0.001$ \\
\hline \multicolumn{3}{|c|}{ A1c mean $(\%)($ ref $=7-7.9)$} \\
\hline$<6$ & 0.67 (0.56 to 0.79$)$ & $<0.001$ \\
\hline $6-6.9$ & 0.79 (0.71 to 0.87$)$ & $<0.001$ \\
\hline $8-8.9$ & 1.09 (0.98 to 1.22$)$ & 0.115 \\
\hline$\geq 9$ & 1.48 (1.29 to 1.70$)$ & $<0.001$ \\
\hline
\end{tabular}

${ }^{*}$ One patient was dropped from logistic regression due to missing service-connected disability.

$\dagger$ Each model was run with a measure of $A 1 c$ variability in quartiles, A1c mean, and the covariates listed in the Methods section. $\mathrm{HRH}$, hypoglycemia-related hospitalization; ref, reference.

copayments for VA services), and percentage of serviceconnected disability (as a marker of disability status, where $>50 \%$ exempts patients from copayments). Other clinical covariates from the baseline period included glucose-lowering medications (eg, insulin, sulfonylurea, metformin, alpha-glucosidase inhibitor, thiazolidinedione, and less commonly used medications), serum creatinine, urine albumin to creatinine ratio, serum albumin, and BMI. All biological measures were averaged over the baseline period. We calculated the logarithmic number of outpatient and inpatient visits from the baseline period to account for utilization of clinical services.
Year of follow-up was included to account for secular changes in diabetes management over time.

\section{Statistical analysis}

Statistical analyses were performed using STATA MP V.15.1. Patient characteristics in the HRH and non-HRH populations were assessed for significance with the $\chi^{2}$ test for binary attributes, the Wilcoxon rank-sum test for intervals of clinical characteristics, and the two-sample t-test for continuous measures. We performed a logistic regression for each A1c variability measure to evaluate the relationship between A1c variability and the risk of HRH in the 2-year follow-up period, controlling for relevant clinical and sociodemographic covariates. Results were expressed as OR with their 95\% CI. A p value of less than 0.05 was considered statistically significant.

\section{Sensitivity analyses}

To test the robustness of our results, we evaluated statistical models with 1-year and 3-year follow-up periods. Because prior HRH may confer higher risk for new HRH events, ${ }^{8}$ we evaluated the association between A1c variability and HRH risk with an additional covariate that identified patients with any HRH during the baseline period. We also determined if the number of A1c tests during the baseline period impacted the study results.

\section{RESULTS}

\section{Study cohort}

The study sample of 342059 had 2871 patients with one or more HRH in the 2-year follow-up period. The baseline sociodemographic and clinical characteristics of patients with no HRH and those who developed HRH in the 2-year follow-up period are presented in table 1. Both groups were predominantly male and white, but the HRH group had twice the percentage of black patients than the non-HRH group. The average (SD) age of patients in the HRH and non-HRH groups was 75.8 (5.5) and 74.1 (5.5) years, respectively, and the average (SD) A1c level was $7.5 \%(1.2 \%)(58 \mathrm{mmol} / \mathrm{mol})$ and $7.0 \%(1.0 \%)$ $(53 \mathrm{mmol} / \mathrm{mol})$, respectively. Insulin, sulfonylurea, and thiazolidinedione use was higher and metformin use was lower in the HRH group. There were more patients with A1c $\geq 9 \%$ ( $75 \mathrm{mmol} / \mathrm{mol})$ in the $\mathrm{HRH}$ population, whereas in the non-HRH population there were more patients with A1c $\leq 7 \%(53 \mathrm{mmol} / \mathrm{mol})$. The mean values of A1c CV, A1c SD, and adjusted A1c SD (10\%, 0.76, and 1.72 , respectively) were significantly higher $(\mathrm{p}<0.001)$ among patients with HRH than those without HRH $(7 \%$, 0.54 , and 1.28).

There was a consistent and positive relationship between A1c variability and HRH in models that controlled for mean A1c levels and sociodemographic and clinical covariates (table 2). A1c CV, A1c SD, and adjusted A1c SD showed increasing risk of HRH throughout quartiles 2-4 in comparison with quartile 1 . The adjusted A1c SD had significantly increased odds of $\mathrm{HRH}$ in the highest quartile. Higher mean A1c levels were also associated with 
Table 3 A1c variability and HRH risk in 1-year and 3-year follow-up periods

\begin{tabular}{|c|c|c|c|c|}
\hline \multirow[b]{2}{*}{ Model† } & \multicolumn{2}{|l|}{1 -year $(n=375519)$} & \multicolumn{2}{|l|}{ 3-year ( $n=308241)$} \\
\hline & OR $(95 \% \mathrm{Cl})$ & $P$ value & OR $(95 \% \mathrm{Cl})$ & $P$ value \\
\hline \multicolumn{5}{|l|}{ Model 1} \\
\hline \multicolumn{5}{|c|}{ A1c coefficient of variation (\%) $(r e f<4)$} \\
\hline $4-5.9$ & 1.10 (0.92 to 1.31$)$ & 0.286 & 1.20 (1.06 to 1.35$)$ & 0.003 \\
\hline $6-9.4$ & 1.12 (0.94 to 1.33$)$ & 0.201 & 1.35 (1.20 to 1.52$)$ & $<0.001$ \\
\hline $9.5-66$ & 1.33 (1.12 to 1.58$)$ & 0.001 & $1.48(1.31$ to 1.67$)$ & $<0.001$ \\
\hline \multicolumn{5}{|l|}{ Model 2} \\
\hline \multicolumn{5}{|c|}{ A1c SD (ref <0.25) } \\
\hline $0.25-0.40$ & $1.18(0.98$ to 1.41$)$ & 0.075 & 1.26 (1.11 to 1.43$)$ & $<0.001$ \\
\hline $0.41-0.68$ & 1.15 (0.96 to 1.38$)$ & 0.138 & $1.42(1.25$ to 1.60$)$ & $<0.001$ \\
\hline $0.69-6.46$ & 1.40 (1.16 to 1.69$)$ & 0.001 & 1.58 (1.39 to 1.80$)$ & $<0.001$ \\
\hline \multicolumn{5}{|l|}{ Model 3} \\
\hline \multicolumn{5}{|c|}{ Adjusted A1c SD (ref <0.62) } \\
\hline $0.62-0.97$ & $1.02(0.86$ to 1.21$)$ & 0.812 & 1.17 (1.04 to 1.32$)$ & 0.008 \\
\hline $0.98-1.56$ & 1.03 (0.87 to 1.22$)$ & 0.731 & 1.23 (1.10 to 1.38$)$ & 0.001 \\
\hline $1.57-17.40$ & 1.29 (1.09 to 1.54$)$ & 0.004 & $1.48(1.31$ to 1.67$)$ & $<0.001$ \\
\hline
\end{tabular}

${ }^{*}$ The risk of $\mathrm{HRH}$ in 1-year and 3-year follow-up periods was assessed separately using the logistic regression model indicated in the Methods section.

†Each model was run with a measure of A1c variability in quartiles, A1c mean, and the covariates listed in the Methods section.

$\mathrm{HRH}$, hypoglycemia-related hospitalization; ref, reference.

greater HRH risk after controlling for each of the Alc variability measures. Compared with patients with mean baseline A1c 7\%-7.9\% (53-63 mmol/mol), patients with Alc $<7 \%(53 \mathrm{mmol} / \mathrm{mol})$ had a significantly lower risk of $\mathrm{HRH}$ and A1c $>9 \%(75 \mathrm{mmol} / \mathrm{mol})$ had a significantly higher risk. Other factors carrying increased HRH risk included insulin and sulfonylurea use, increased urine albumin to creatinine excretion $(>30 \mathrm{mg} / \mathrm{g})$, higher serum creatinine $(>1.2 \mathrm{mg} / \mathrm{dL})$, black race, and age $\geq 75$ years. These associations remained consistent across all three measures of A1c variability (online supplemental appendix tables 1-3).

\section{Sensitivity analyses}

The same models of A1c CV, A1c SD and adjusted A1c SD were used to study 1-year and 3-year follow-up periods (table 3; online supplemental appendix tables 4 and 5). During the 1-year of follow-up, relationships between A1c variability measures and $\mathrm{HRH}$ risk were significant in the highest quartile. The 3-year follow-up model generated ORs very similar to the 2-year model, showing increased HRH risk associated with all Alc variability measures.

Additional analysis that assessed the impact of prior HRH events did not modify the association between A1c variability and increased HRH risk (table 4). Prior HRH conferred a threefold higher risk of future $\mathrm{HRH},{ }^{8}$ but higher A1c variability and mean A1c continued to be significantly associated with HRH (table 4; online supplemental appendix tables 6-8).

To determine if more frequent A1c testing during baseline impacted the study results, we included the number of A1c tests in the analysis model of A1c CV. This did not change the study results (data not shown).

\section{DISCUSSION}

We found a significant and positive relationship between higher A1c variability and HRH over a 2-year follow-up period among veterans with diabetes who were 65 years or older. A1c levels and variability were measured over a 3-year baseline period and patients were then followed to assess HRH events. Significance of the associations and the level of risk varied somewhat across the different A1c variability measures, but all showed consistent and graded relationships with HRH. Average A1c levels were also significantly and independently associated with $\mathrm{HRH}$, with levels $<7.0 \%$ (53 $\mathrm{mmol} / \mathrm{mol})$ associated with lower risk and levels $>9 \%(75 \mathrm{mmol} / \mathrm{mol})$ conferring greater risk. In sensitivity analyses, prior HRH carried higher HRH risk, but when prior HRH was included as a covariate, A1c variability measures remained strong predictors of HRH. High Alc variability was significantly and independently associated with risk of HRH for up to 3 years following the baseline period.

Clinical practice guidelines ${ }^{13-15}$ have emphasized the need for individualized and higher A1c targets in older adults with diabetes to balance risks and benefits. Our results also suggest that A1c variability has an independent and significant effect on HRH risk, and tracking Alc levels alone may be insufficient to mitigate risk. We confirmed that guideline-directed A1c targets for many older adults with diabetes are reasonable for minimizing 
Table 4 Sensitivity analysis of prior HRH's impact on HRH risk* $^{*}(n=342$ 058)†

\begin{tabular}{|c|c|c|}
\hline Modelł & OR $(95 \% \mathrm{Cl})$ & $P$ value \\
\hline \multicolumn{3}{|l|}{ Model 1} \\
\hline \multicolumn{3}{|c|}{ A1c coefficient of variation $(\%)($ ref <4) } \\
\hline $4-5.9$ & 1.19 (1.04 to 1.37$)$ & 0.010 \\
\hline $6-9.4$ & $1.28(1.12$ to 1.46$)$ & $<0.001$ \\
\hline $9.5-66$ & $1.42(1.24$ to 1.63$)$ & $<0.001$ \\
\hline \multicolumn{3}{|c|}{ Prior HRH (ref=no) } \\
\hline Yes & 3.12 (2.65 to 3.67$)$ & $<0.001$ \\
\hline \multicolumn{3}{|c|}{ A1c mean $(\%)(r e f=7-7.9)$} \\
\hline$<6$ & 0.67 (0.56 to 0.79$)$ & $<0.001$ \\
\hline $6-6.9$ & $0.78(0.71$ to 0.86$)$ & $<0.001$ \\
\hline $8-8.9$ & $1.11(1.00$ to 1.25$)$ & 0.057 \\
\hline$\geq 9$ & 1.53 (1.33 to 1.75$)$ & $<0.001$ \\
\hline \multicolumn{3}{|l|}{ Model 2} \\
\hline \multicolumn{3}{|c|}{ A1c SD (ref <0.25) } \\
\hline $0.25-0.40$ & 1.26 (1.10 to 1.45$)$ & 0.001 \\
\hline $0.41-0.68$ & 1.35 (1.17 to 1.55$)$ & $<0.001$ \\
\hline $0.69-6.46$ & 1.54 (1.33 to 1.79$)$ & $<0.001$ \\
\hline \multicolumn{3}{|c|}{ Prior $\mathrm{HRH}(\mathrm{ref}=\mathrm{no})$} \\
\hline Yes & 3.12 (2.65 to 3.67$)$ & $<0.001$ \\
\hline \multicolumn{3}{|c|}{ A1c mean $(\%)(r e f=7-7.9)$} \\
\hline$<6$ & 0.71 (0.60 to 0.85$)$ & $<0.001$ \\
\hline $6-6.9$ & 0.81 (0.73 to 0.89$)$ & $<0.001$ \\
\hline $8-8.9$ & 1.09 (0.98 to 1.23$)$ & 0.117 \\
\hline$\geq 9$ & 1.49 (1.30 to 1.72$)$ & $<0.001$ \\
\hline \multicolumn{3}{|l|}{ Model 3} \\
\hline \multicolumn{3}{|c|}{ Adjusted A1c SD (ref <0.62) } \\
\hline $0.62-0.97$ & 1.08 (0.95 to 1.23$)$ & 0.253 \\
\hline $0.98-1.56$ & 1.11 (0.97 to 1.26$)$ & 0.127 \\
\hline $1.57-17.40$ & 1.36 (1.19 to 1.56$)$ & $<0.001$ \\
\hline \multicolumn{3}{|c|}{ Prior HRH (ref=no) } \\
\hline Yes & 3.12 (2.65 to 3.68 ) & $<0.001$ \\
\hline \multicolumn{3}{|c|}{ A1c mean (\%) (ref=7-7.9) } \\
\hline$<6$ & 0.67 (0.57 to 0.80$)$ & $<0.001$ \\
\hline $6-6.9$ & $0.79(0.71$ to 0.87$)$ & $<0.001$ \\
\hline $8-8.9$ & 1.10 (0.98 to 1.23$)$ & 0.113 \\
\hline$\geq 9$ & 1.48 (1.29 to 1.70$)$ & $<0.001$ \\
\hline
\end{tabular}

*The sensitivity analysis was executed by adding prior HRH from the baseline period as a binary covariate to the logistic regression indicated in the Methods section.

†One patient was dropped from logistic regression due to missing service-connected disability.

‡Each model was run with a measure of $A 1 c$ variability in quartiles, previous $\mathrm{HRH}$ event, $\mathrm{A} 1 \mathrm{c}$ mean, and the covariates listed in the Methods section.

$\mathrm{HRH}$, hypoglycemia-related hospitalization; ref, reference.

risk of $\mathrm{HRH}$, since Alc levels between $7 \%$ and $8.9 \%$ (53-74 mmol/mol) carried similar risk. We also showed that A1c levels $>9 \%(75 \mathrm{mmol} / \mathrm{mol})$ are linked to increased risk of $\mathrm{HRH}$ and lower levels $(<7 \%, 53 \mathrm{mmol} / \mathrm{mol})$ are associated with lower risk. Studies have shown differing relationships between Alc levels and severe hypoglycemia, with high A1c, ${ }^{1629}$ low A1c, ${ }^{17}$ or both ${ }^{30}$ carrying increased risk. ${ }^{16} 1730$ Unlike other studies, we included a large and broad sample of older adults with diabetes and captured outcomes from both VA and Medicare data. It is possible that differences across various studies may reflect variations in the patient population, diabetes treatment, definitions of hypoglycemia, and duration of follow-up. We acknowledge that several methods for calculating Alc variability have been proposed, including traditional variance measures such as $\mathrm{CV}$ and $\mathrm{SD}$, as well as categorical measures that incorporate absolute change in A1c. ${ }^{18} 19232531$ Since the majority of prior publications have used CV or SD to measure Alc variability we also opted for these methods.

Additional significant risk factors associated with HRH include use of insulin or sulfonylurea medications, black race, elevated serum creatinine, increased urine albumin to creatinine ratio, and age $>75$ years. Many of these same characteristics or conditions have been associated with risk of severe hypoglycemia. ${ }^{7-10}$ It is most likely that these factors are linked to HRH through effects of treatment, including adverse effects, or are markers of disease burden. Prior HRH events were also significantly associated with future risk of $\mathrm{HRH}$, as has been previously shown. ${ }^{8} 18$ Metformin usage and high BMI were associated with lower risk of HRH. Metformin has been associated with lower incidence of hypoglycemia ${ }^{32}$ and higher BMI has been shown to carry reduced incidence of severe hypoglycemia, possibly due to the increased insulin resistance present in obesity. ${ }^{33-35}$

Patients at highest risk for HRH are those with both high A1c levels and high A1c variability, and these clinical findings often reflect the complex interplay of disease severity, treatment, and sociodemographic factors. For example, patients with high A1c levels and A1c variability are more likely to be treated with insulin or multidrug regimens, have competing conditions or comorbidities that complicate diabetes treatment, ${ }^{31} 36$ and experience medication adherence issues. ${ }^{37}$ Alc variability is clearly influenced by these underlying factors that affect glucose control over time. The fact that increasing variability is independently associated with HRH should not be overlooked as a marker of increased risk. From an implementation standpoint, healthcare systems may choose to calculate A1c variability measures and identify patients at high risk for major hypoglycemia events. A1c CV $\geq 6 \%$, A1c SD $>0.4$ and A1c $>9 \%$ identify patients at increased HRH risk over a period of 2-3years. The presence of these measures may alert physicians to individualize care and minimize such risks.

Our study has limitations that may affect its generalizability. The study sample represented an older and predominantly white male population and we included only patients with at least four A1c levels over 3 years. Further, the study sample included only veterans, which is a group that has a high prevalence of diabetes, ${ }^{26}$ has greater physical and 
mental health comorbidities relative to the general population, ${ }^{38}$ and may have a substantial number of patients who are potentially overtreated. ${ }^{11}$ Our results may not extend to younger patients or those with fewer comorbidities. Limited data were available on socioeconomic status and this may not fully account for the impact of social determinants of health on HRH outcomes. We assessed HRH as our outcome of interest, although this represents a more severe form of hypoglycemia. Administrative data do not reliably include milder forms of hypoglycemia such as those treated in the outpatient setting, so these more frequent events were not captured. In addition, our findings do not allow us to determine causality. Nonetheless, the study design has several strengths. We employed a large study sample encompassing a 12-year study period and employed various A1c variability measures. We applied a 3-year baseline period before determining HRH outcomes, which limits concerns about reverse causality. Statistical models included a number of relevant covariates, and we performed sensitivity analyses to assess the robustness of the findings.

In summary, older adults with diabetes with increasing Alc variability and elevated A1c levels $(>9 \%, 75 \mathrm{mmol} /$ mol) are at significantly greater risk of HRH over a 2-year period. Our results suggest that clinicians should consider A1c variability for its potential role in predicting risk of severe hypoglycemia.

\section{Author affiliations}

${ }^{1}$ Center for Healthcare Organization and Implementation Research (CHOIR), VA Boston Health Care System, Boston, Massachusetts, USA

${ }^{2}$ Boston University School of Medicine, Boston, Massachusetts, USA

${ }^{3}$ Psychiatry, Boston University School of Medicine, Boston, Massachusetts, USA

${ }^{4}$ Health Law, Policy and Management, Boston University School of Public Health, Boston, Massachusetts, USA

${ }^{5}$ Medical Service (111), VA Boston Healthcare System, West Roxbury,

Massachusetts, USA

${ }^{6}$ Harvard Medical School, Boston, Massachusetts, USA

\section{Twitter David C Mohr @david_c_mohr}

Contributors All of the authors made substantial contributions to the intellectual content of the paper, including the design of the study (all authors), acquisition of data (MJYZ), statistical analyses (MJYZ, DCM), interpretation of data (all authors), and drafting and critical revision of the manuscript (all authors). MJYZ is the guarantor of this work and as such had full access to all the data in the study and takes responsibility for the integrity of the data and the accuracy of the data analysis.

Funding The study was supported by the Department of Veterans Affairs, Veterans Health Administration, Office of Research and Development, Health Services Research and Development (IIR 15-116) and the National Institutes of Health, National Institute of Diabetes and Digestive and Kidney Diseases (R01 DK114098). These funding agencies had no role in study design, data analysis, manuscript preparation or the decision to submit for publication.

Disclaimer The views expressed in this article are those of the authors and do not necessarily reflect the position or policy of the Department of Veterans Affairs or the US government. This work was produced by employees of the US government as part of his/her official duties. No copyright exists and therefore it cannot be transferred.

Competing interests None declared.

Patient consent for publication Not required.

Ethics approval The study was reviewed and approved by the institutional review board of the VA Boston Healthcare System.
Provenance and peer review Not commissioned; externally peer reviewed.

Data availability statement Data are available upon reasonable request. All data relevant to the study are included in the article or uploaded as supplemental information.

Supplemental material This content has been supplied by the author(s). It has not been vetted by BMJ Publishing Group Limited (BMJ) and may not have been peer-reviewed. Any opinions or recommendations discussed are solely those of the author(s) and are not endorsed by BMJ. BMJ disclaims all liability and responsibility arising from any reliance placed on the content. Where the content includes any translated material, BMJ does not warrant the accuracy and reliability of the translations (including but not limited to local regulations, clinical guidelines, terminology, drug names and drug dosages), and is not responsible for any error and/or omissions arising from translation and adaptation or otherwise.

Open access This is an open access article distributed in accordance with the Creative Commons Attribution Non Commercial (CC BY-NC 4.0) license, which permits others to distribute, remix, adapt, build upon this work non-commercially, and license their derivative works on different terms, provided the original work is properly cited, appropriate credit is given, any changes made indicated, and the use is non-commercial. See: http://creativecommons.org/licenses/by-nc/4.0/.

ORCID ID

Molly J Y Zhao http://orcid.org/0000-0002-2457-3108

\section{REFERENCES}

1 Abdelhafiz AH, Sinclair AJ. Cognitive frailty in older people with type 2 diabetes mellitus: the central role of hypoglycaemia and the need for prevention. Curr Diab Rep 2019;19:15.

2 Feil DG, Rajan M, Soroka O, et al. Risk of hypoglycemia in older veterans with dementia and cognitive impairment: implications for practice and policy. J Am Geriatr Soc 2011;59:2263-72.

3 Kirsh SR, Aron DC. Choosing targets for glycaemia, blood pressure and low-density lipoprotein cholesterol in elderly individuals with diabetes mellitus. Drugs Aging 2011;28:945-60.

4 Whitmer RA, Karter AJ, Yaffe K, et al. Hypoglycemic episodes and risk of dementia in older patients with type 2 diabetes mellitus. JAMA 2009;301:1565-72.

5 Zoungas S, Patel A, Chalmers J, et al. Severe hypoglycemia and risks of vascular events and death. N Engl J Med 2010;363:1410-8.

6 Lipska KJ, Ross JS, Wang Y, et al. National trends in US hospital admissions for hyperglycemia and hypoglycemia among Medicare beneficiaries, 1999 to 2011. JAMA Intern Med 2014;174:1116-24.

7 Cheng PC, Hsu SR, Tu ST, et al. Body mass index influences the plasma glucose concentration during iatrogenic hypoglycemia in people with type 2 diabetes mellitus: a cross-sectional study. PeerJ 2018;6:e4348.

8 Karter AJ, Warton EM, Lipska KJ, et al. Development and validation of a tool to identify patients with type 2 diabetes at high risk of Hypoglycemia-Related emergency department or hospital use. JAMA Intern Med 2017;177:1461-70.

9 Lee AK, Lee CJ, Huang ES, et al. Risk factors for severe hypoglycemia in black and white adults with diabetes: the Atherosclerosis risk in communities (ARIC) study. Diabetes Care 2017;40:1661-7.

10 Yun J-S, Ko S-H, Ko S-H, et al. Presence of macroalbuminuria predicts severe hypoglycemia in patients with type 2 diabetes: a 10year follow-up study. Diabetes Care 2013;36:1283-9.

11 Tseng C-L, Soroka O, Maney M, et al. Assessing potential glycemic overtreatment in persons at hypoglycemic risk. JAMA Intern Med 2014;174:259-68.

12 Handelsman Y, Bloomgarden ZT, Grunberger G, et al. American association of clinical endocrinologists and american college of endocrinology - clinical practice guidelines for developing a diabetes mellitus comprehensive care plan - 2015. Endocr Pract 2015;21 Suppl 1:1-87.

13 American Diabetes Association. 6. Glycemic Targets: Standards of Medical Care in Diabetes-2020. Diabetes Care 2020;43:S66-76.

14 LeRoith D, Biessels GJ, Braithwaite SS, et al. Treatment of diabetes in older adults: an endocrine Society* clinical practice guideline. $J$ Clin Endocrinol Metab 2019;104:1520-74.

15 Conlin PR, Colburn J, Aron D, et al. Synopsis of the 2017 U.S. department of Veterans Affairs/U.S. Department of defense clinical practice guideline: management of type 2 diabetes mellitus. Ann Intern Med 2017;167:655-63.

16 Miller ME, Bonds DE, Gerstein HC, et al. The effects of baseline characteristics, glycaemia treatment approach, and glycated haemoglobin concentration on the risk of severe hypoglycaemia: 
post hoc epidemiological analysis of the ACCORD study. BMJ 2010;340: :b5444.

17 Yu S, Fu AZ, Engel SS, et al. Association between hypoglycemia risk and hemoglobin $\mathrm{A} 1 \mathrm{C}$ in patients with type 2 diabetes mellitus. Curr Med Res Opin 2016;32:1409-16.

18 Critchley JA, Carey IM, Harris T, et al. Variability in glycated hemoglobin and risk of poor outcomes among people with type 2 diabetes in a large primary care cohort study. Diabetes Care 2019;42:2237-46.

19 Forbes A, Murrells T, Mulnier $\mathrm{H}$, et al. Mean $\mathrm{HbA}_{1}, \mathrm{HbA}_{10}$ variability, and mortality in people with diabetes aged 70 years and older: a retrospective cohort study. Lancet Diabetes Endocrinol 2018;6:476-86.

20 Gorst C, Kwok CS, Aslam S, et al. Long-Term glycemic variability and risk of adverse outcomes: a systematic review and metaanalysis. Diabetes Care 2015;38:2354-69.

21 Prentice JC, Pizer SD, Conlin PR. Identifying the independent effect of $\mathrm{HbA}_{1 \mathrm{c}}$ variability on adverse health outcomes in patients with Type 2 diabetes. Diabet Med 2016;33:1640-8.

22 Wan EYFet al. Age-Specific associations of $\mathrm{HbA1c}$ variability with cardiovascular disease and mortality in type 2 diabetes mellitus patients: a 10-year cohort study. Diabetes Obes Metab 2020.

23 Zhong VW, Juhaeri J, Cole SR, et al. $\mathrm{HbA}_{1 \mathrm{C}}$ variability and hypoglycemia hospitalization in adults with type 1 and type 2 diabetes: A nested case-control study. J Diabetes Complications 2018;32:203-9.

24 Hirakawa $\mathrm{Y}$, Arima $\mathrm{H}$, Zoungas S, et al. Impact of visit-to-visit glycemic variability on the risks of macrovascular and microvascular events and all-cause mortality in type 2 diabetes: the advance trial. Diabetes Care 2014;37:2359-65.

25 Sheng C-S, Tian J, Miao Y, et al. Prognostic Significance of Longterm $\mathrm{HbA}_{1 \mathrm{c}}$ Variability for All-Cause Mortality in the ACCORD Trial. Diabetes Care 2020;43:1185-90.

26 Miller DR, Safford MM, Pogach LM. Who has diabetes? Best estimates of diabetes prevalence in the Department of Veterans Affairs based on computerized patient data. Diabetes Care 2004;27:B10-21.
27 Ginde AA, Blanc PG, Lieberman RM, et al. Validation of ICD-9-CM coding algorithm for improved identification of hypoglycemia visits. BMC Endocr Disord 2008;8:4.

28 Karter AJ, Warton EM, Moffet $\mathrm{HH}$, et al. Revalidation of the hypoglycemia risk stratification tool using ICD-10 codes. Diabetes Care 2019;42:e58-9.

29 Williams ME, Garg R, Wang W, et al. High hemoglobin A1c levels and glycemic variability increase risk of severe hypoglycemia in diabetic hemodialysis patients. Hemodial Int 2014;18:423-32.

30 Lipska KJ, Warton EM, Huang ES, et al. HbA1c and risk of severe hypoglycemia in type 2 diabetes: the diabetes and aging study. Diabetes Care 2013;36:3535-42.

31 Orsi E, Solini A, Bonora E, et al. Haemoglobin A1c variability is a strong, independent predictor of all-cause mortality in patients with type 2 diabetes. Diabetes Obes Metab 2018;20:1885-93.

32 Bolen S, Feldman L, Vassy J, et al. Systematic review: comparative effectiveness and safety of oral medications for type 2 diabetes mellitus. Ann Intern Med 2007;147:386-99.

33 Bodmer M, Meier C, Krähenbühl S, et al. Metformin, sulfonylureas, or other antidiabetes drugs and the risk of lactic acidosis or hypoglycemia: a nested case-control analysis. Diabetes Care 2008;31:2086-91.

34 Khunti K, Alsifri S, Aronson R, et al. Rates and predictors of hypoglycaemia in 27585 people from 24 countries with insulintreated type 1 and type 2 diabetes: the global HAT study. Diabetes Obes Metab 2016;18:907-15.

35 Home P, Calvi-Gries F, Blonde L, et al. Clinical correlates of hypoglycaemia over 4 years in people with type 2 diabetes starting insulin: an analysis from the credit study. Diabetes Obes Metab 2018;20:921-9.

36 Huang ES, Liu JY, Moffet HH, et al. Glycemic control, complications, and death in older diabetic patients: the diabetes and aging study. Diabetes Care 2011;34:1329-36.

37 Rozenfeld Y, Hunt JS, Plauschinat C, et al. Oral antidiabetic medication adherence and glycemic control in managed care. Am J Manag Care 2008;14:71-5.

38 Agha Z, Lofgren RP, VanRuiswyk JV, et al. Are patients at Veterans Affairs medical centers sicker? A comparative analysis of health status and medical resource use. Arch Intern Med 2000;160:3252-7. 